\title{
Faire la Caraïbe, comme on refait le monde
}

\section{Michel Giraud}

\section{(2) OpenEdition \\ Journals}

Édition électronique

URL : http://journals.openedition.org/plc/252

DOI : $10.4000 /$ plc. 252

ISSN : 2117-5209

\section{Éditeur}

L'Harmattan

Édition imprimée

Date de publication : 14 janvier 2004

Pagination : 81-108

ISBN : 2-7475-7061-4

ISSN : $1279-8657$

Référence électronique

Michel Giraud, «Faire la Caraïbe, comme on refait le monde », Pouvoirs dans la Caraïbe [En ligne],

14 | 2004, mis en ligne le 02 mars 2011, consulté le 20 avril 2019. URL : http://

journals.openedition.org/plc/252; DOI : 10.4000/plc.252 


\title{
FAIRe la CARAÏBE, COMME ON REFAIT LE MONDE
}

\author{
par Michel GIRAUD \\ Chercheur CNRS \\ Membre du CRPLC \\ Université des Antilles et de la Guyane
}

"La notion d'antillanité surgit d'une réalité que nous aurons à interroger, mais correspond aussi à un vou dont il nous faudra préciser ou fonder la légitimité "

Edouard Glissant ${ }^{*}$

\section{UN ESPACE FRAGMENTE}

La Caraïbe est une aire fragmentée par de multiples séparations, de diverses sortes. Dans le cas de l'archipel qui en constitue l'épine dorsale, cette fragmentation est particulièrement marquée du fait de la géographie et de l'histoire. Ainsi, l'exiguïté de la plupart des îles de cet archipel (dont on pourrait s'attendre à ce qu'elle pousse leurs habitants à chercher par tous les moyens à élargir leur horizon) et la fréquente proximité de chacune d'elles avec ses voisines (au point que souvent des côtes de l'une on peut apercevoir les rivages des autres), une proximité qui met l'élargissement envisagé à la portée de ceux qui veulent l'entreprendre, n'ont cependant que fort peu concouru à contrecarrer l'éclatement de la zone en question par le développement de relations nombreuses entre les différentes populations habitant celle-ci. C'est que l'ont emporté les oppositions héritées du partage colonial de cette zone entre diverses métropoles comme les divisions entre les possessions voisines d'une même métropole ${ }^{1}$, de même que la segmentation en

\footnotetext{
${ }^{*}$ Le discours antillais, Paris, Editions du Seuil, 1981, p. 422.

${ }^{1}$ "From the very beginning, although they were placed in the same colonial situation, the neighboring islands of the Leewards never cooperated, but "each island preferred to go its own way, concentrating its attention upon its own separate existence and its own separate institutions" (Elsa Goveia, Slave Society in the British Leeward Islands at the End of the Eighteenth Century, New Haven, Yale University Press, 1965, p. 71,81). This refers initially to the white planters, but clearly if the whites of neighboring islands never got together, it is doubtful that they would allow their slaves to do so. Thus from
} 
groupes socio-raciaux relativement étanches qui a accompagné la colonisation de chacun des pays de cette région, au point que certains d'entre eux ont été décrits - sous le vocable de sociétés plurales - comme le lieu de la fragmentation sociale par excellence.

Comme on le sait bien, le partage dont il vient d'être question a entraîné dans la région une forte polarisation des échanges, dont le régime dit de l'Exclusif ${ }^{2}$ a longtemps été l'archétype. Ainsi, concernant chacune des îles de l'archipel caraíbe, c'est avec un pays lointain situé hors de la région (l'ancienne métropole coloniale, l'actuelle puissance tutélaire ou encore le pays de réception d'un grand nombre d'enfants de cette île, partis comme émigrants) que se fait - en une dyade étroite l'essentiel des différents types d'échanges (voir Burac, 1995, pp. 102106 ), et non pas avec l'environnement immédiat.

Certes, depuis plusieurs décennies, des tentatives d'unifier la Caraïbe se sont fait jour à différents niveaux, comme par exemple, au plan de l'économie et du commerce, à travers le Marché commun de la Caraíbe (CARICOM), ou en matière de culture, à travers la Carifesta, ou enfin, plus récemment, pour ce qui est des relations politiques, à travers l'Association des Etats de la Caraïbe. Et des liens de nature diverse (échanges scolaires, universitaires et sportifs, tournées d'orchestres, déplacements touristiques, etc.) tendent quelque peu à se développer entre les habitants de la Caraỉbe insulaire, qui commencent à découvrir les réalités de leur «cour intérieure ». Mais ces relations restent d'une ampleur et, surtout, d'une portée limitées et les projets d'unification plus ambitieux ont échoué, comme par exemple celui, déjà ancien, qui devait associer les pays de la Caraïbe anglophone dans une Federation of the

earliest times they developed a sense of insularity, a sense of island identity, a trait which most observers and present events constantly confirm" (Wilson, 1973, pp. 190191).

${ }^{2}$ Il s'agit du régime commercial imposant que la Métropole soit l'unique fournisseur et l'unique client de la colonie et que les importations comme les exportations de cette dernière ne se fassent que par des navires battant pavillon métropolitain. 
West Indies ${ }^{3}$. De plus, les relations qui, dans un passé plus ou moins lointain, ont existé entre différents pays de la région (fuite d'esclaves passant d'une île à une autre, anciennes migrations intracaraïbéennes de travailleurs et de leurs familles, etc.) ne semblent pas avoir laissé de traces significatives dans la mémoire collective de ces pays: par exemple, qui se souvient aux Antilles françaises qu'un nombre non négligeable de Guadeloupéens et de Martiniquais sont partis travailler à la construction du canal de Panama, puis de là sur les plantations bananières au Costa Rica, ou que plusieurs familles guadeloupéennes sont allées s'installer un temps en Haïti, certaines y prenant souche?

\section{UNE UNITE A CONSTRUIRE}

Contrastant avec ce qui nous parait être l'absence d'un sentiment marqué d'appartenance à un même ensemble, il y a déjà bien longtemps que des analyses de caractère scientifique ont identifié la Caraïbe comme une aire présentant objectivement une certaine unité au plan culturel. Une unité qui - au-delà des régularités frappantes à travers lesquelles elle se manifeste au plan de l'organisation sociale (types d'organisation et de relations familiales, par exemple), de la culture matérielle (techniques de production et modes d'alimentation) ou des productions symboliques et artistiques (croyances et pratiques magico-religieuses, littérature orale et expressions musicales) - tient à la présence, partout attestée dans la région, non pas d'une entité culturelle unique (les cultures créoles sont multiples dans leur forme) mais d'un même processus de fabrication culturelle - celui de la créolisation ${ }^{4}$ - dont les différentes cultures créoles

\footnotetext{
${ }^{3}$ La sécession de l'île d'Aruba de la Fédération des Antilles néerlandaises, qui n'a pas pour autant mis fin à l'appartenance de cette île au Royaume des Pays-Bas, témoigne, pour la Caraïbe de colonisation hollandaise, du même type d'échec.

${ }^{4}$ La créolisation désignant au sens strict un processus général de synthèse culturelle et non une identité (une culture) particulière, l'analyse scientifique ne peut que considérer comme fautif l'usage politico-idéologique qui est parfois fait de cette notion (notamment dans les sociétés plurales de la Caraỉbe), lorsqu'elle ne renvoie qu'à la seule identité des "Afro-créoles» et qu'alors, comme l'écrit Ralph Premdas, "creolization is a hegemonic concept that elevates the cultural practices of one community as the measure of membership and entitlement [...] a threat of
} 
sont autant de produits. Soit un processus de réinterprétation d'éléments et de modèles culturels importés des quatre coins du monde en vue de leur adaptation à un nouvel environnement physique et social. Un processus unique qui - renvoyant aux dynamiques historiques de l'esclavage de "nègres » amenés d'Afrique, puis du travail sous contrat d'immigrants asiatiques, qui ont fait de la plupart des pays de la région des "sociétés de plantation $"^{5}$ - donne naissance, par synthèse, à des mélanges inédits, ne pouvant être réduits ni à un quelconque de leurs ingrédients ni à la simple addition de ceux-ci, et qui, parce que ces dynamiques ont été diverses, a fait émerger des cultures créoles différentes.

Cependant, le fait que l'on puisse distinguer objectivement, «à froid », «sur le papier » dirait Pierre Bourdieu (voir Bourdieu, 1987), un ensemble caraibéen dans le grand atlas des aires culturelles du monde ne suffit pas, malgré toute la légitimité que confère l'appui d'analyses scientifiques, à affirmer l'existence en acte, «à chaud», d'une communauté caraíbéenne ${ }^{6}$. Tant il est vrai qu'il ne peut pas y avoir de communauté à proprement parler sans que celle-ci ne soit subjectivement éprouvée, ressentie et même affirmée, sans donc une conscience et une affirmation d'appartenance. Or, même s'il n'est pas douteux qu'un ensemble de traits objectifs partagés peut permettre à l'ethnographie de caractériser, de l'extérieur, la région caraíbe comme constituant une aire culturelle particulière, il ne nous semble pas moins assuré que la faiblesse que nous avons repérée en ce qui concerne le sentiment d'une appartenance commune qu'éprouveraient, de l'intérieur, les populations

homogenization by a hegemonic community attempting to impose its cultural writ on the lives of other » (Premdas, 1996, pp. 14 et 16).

${ }^{5} \mathrm{Ce}$ dernier caractère paraît si décisif dans l'appréhension de l'identité culturelle caraỉbe que lorsque, au tournant des années 1960, l'anthropologie a voulu définir cette aire dont elle était en train de déterminer les contours, elle l'a appelée «l'Amérique des plantations ». Voir, par exemple, Wagley, 1960.

${ }^{6}$ Comme le note Fred Constant, « la proximité (réelle ou supposée) géographique et culturelle de pays appartenant à la même région n'implique aucune vocation naturelle à l'intégration politique ou à la coopération économique ». Voir Constant, 1996b, p. 137. 
de cette région empêche de conclure à l'existence certaine d'une véritable communauté caraïbéenne.

Comme il en va de toute communauté, ce sentiment n'a de chance de se cristalliser - dès lors qu'existent des similitudes objectives qui peuvent constituer la base matérielle d'une telle cristallisation - que si se fait jour une volonté de la part des élites intellectuelles mais aussi sociales et politiques des pays de la région d'affirmer cette dernière comme un espace marqué de ses intérêts propres et prêt à s'approprier son histoire commune pour défendre son originalité face aux projets concurrents de son entour immédiat ou lointain, et en conséquence de construire cette région en tant qu'entité autonome. Soutenir, comme nous le faisons ici, l'idée qu'actuellement la Caraïbe est davantage la représentation d'une volonté encore incertaine qu'une donnée de l'observation, un vou plus qu'un fait et qu'à ce titre elle a un futur - avec ce que comporte d'incertitude cette modalité de la temporalité - plutôt qu'un passé, temps dont la nécessité est toujours la marque, c'est dire que son existence dépend de la formulation et du développement d'un projet politique. C'est aussi souligner plus généralement, contre tout possibilisme, que la politique est affaire de volonté, d'une volonté certes informée par l'analyse de l'existant et limitée par les contraintes que fait peser celui-ci mais qui ne se réduit pas pour autant au seul souci de la meilleure gestion de ce qui existe, puisqu'elle a à tordre l'actuel pour faire advenir le souhaité. Et c'est enfin rappeler en particulier - contre l'opinion de ceux qui tirent du constat de la grande hétérogénéité de la Caraibe la conclusion de l'impossibilité d'une "maison commune" caraíbéenne (voir par exemple, Premdas, 1996) - que les entités régionales existant de par le monde sont avant tout le résultat d'une volonté politique forte davantage que la traduction institutionnelle d'une communauté objective qui aurait préexisté à cette institutionnalisation. Nous en voulons pour preuve la construction contemporaine, aux plans économique et politique, de l'Europe, un ensemble physiquement plus différencié et - concernant tous les facteurs que retient Ralph Premdas dans son analyse citée : le phénotype, la langue, la culture, la religion, la taille de la population des pays qui le composent ou leur niveau de vie- 
au moins tout aussi divers que ne l'est la Caraïbe elle-même. Une région du monde qui, de plus, a été, durant plusieurs siècles, le théâtre de nombreuses guerres entre ses parties constituantes, les guerres les plus meurtrières que l'humanité ait connues, et dont, par ailleurs, la construction politique récente laisse, pour l'instant, en marge des pays qui pourraient «objectivement» tout aussi bien y être impliqués. Une région enfin dont l'unité de fait est si peu marquée que, plutôt qu'à se référer à elle pour justifier la construction politique en question, les promoteurs de celle-ci préfèrent en appeler à une communauté de pensée (celle qui est présentée comme l'héritage de la philosophie des droits de l'Homme) dont, pourtant, l'histoire récente de l'Europe, avec sa kyrielle de régimes politiques autoritaires ou même totalitaires et son cortège de massacres et d'exactions de toutes sortes, nous rappelle à quel point elle n'est qu'imaginée. Pourquoi donc ce qui est en train d'être réussi en Europe serait-il nécessairement et définitivement impossible dans la Caraïbe et ne faut-il pas penser avec Edouard Glissant que face à toute option qui hésite devant «le choix d'antillanité », il faut diagnostiquer « avec certitude un désir camouflé de rester dans les limites assignées par la non-histoire »(Glissant, 1981, p. 423), c'est-à-dire par l'entreprise coloniale?

Il convient maintenant d'indiquer le bénéfice qu'il y aurait à élaborer le projet dont il est question en prenant en compte les grands enseignements qui ressortent de la complexité de l'expérience historique créole.

\section{LE PARADIGME CREOLE}

Toutes les populations de couleur de la Caraïbe ont été arrachées à leurs cultures ancestrales, sans éventualité de retour et, à l'exception des communautés de nègres marrons, sans pouvoir conserver autre chose que des bribes d'une mémoire disloquée de ces cultures. Elles ont été soumises, dans tous les compartiments de leur vie, à l'ordre totalitaire de l'esclavage américain, puis à la loi d'airain de la plantation postesclavagiste. Elles ont donc dû se forger de nouvelles identités sans pouvoir prendre fermement appui sur un arrière-pays culturel préexistant 
à la colonisation, mais en étant totalement placées sous la coupe d'un rapport colonial ici fondateur de toutes choses. Elles l'ont fait en entremêlant, en un formidable foisonnement de syncrétismes, des traditions culturelles fort diverses.

L'identité de ces populations ne pouvant pas s'enraciner dans les cultures anciennes de leurs lointains et divers ancêtres mais procédant de la sublimation d'une situation nouvelle de dépossession et de sujétion, c'est le recours habituel à des généalogies immémoriales pour penser la personnalité profonde des peuples qui se trouve ici empêché. Les sociétés caraibéennes - du fait de la brutalité de la cassure opérée avec la plupart des ascendances de leurs populations - témoignent ainsi fortement d'une impossibilité à fonder l'unité de leurs peuples sur la référence à une quelconque origine, c'est-à-dire à un point zéro, à un commencement absolu, qui se situerait en amont de l'histoire, dans un temps - on le sait bien - qui est celui du mythe. Les peuples de la Carä̈be, s'ils ont bien une histoire, n'ont pas de mythes.

En corollaire de ce qui vient d'être précisé, les dynamiques par lesquelles les identités caraibéennes sont nées du choc, certes brutal mais en définitive fécond, d'ensembles au départ radicalement différents les uns des autres, mettent également en exergue le fait capital que - pour paraphraser une distinction qui a fait école ${ }^{7}$ - ces identités ne relèvent pas de l'intolérance sacrée de la racine mais participent de l'inquiétude roborative de la relation. Et que donc elles ne peuvent être convenablement appréhendées que dans le jeu horizontal des interactions entre les différentes traditions qui sont entrées dans leur composition et certainement pas, nous l'avons dit, dans la référence, en quelque sorte en aplomb, à une ou même plusieurs origines considérées comme fondatrices.

De ce caractère premier de la relation dans la formation de l'identité que manifestent avec éclat les dynamiques en question ressortent fortement, dans les sociétés créoles, la relativité et la labilité

${ }^{7}$ La distinction entre une "identité-racine » et une «identité-relation » proposée par Edouard Glissant. Voir, par exemple, Glissant, 1990. 
des démarcations et des appartenances que l'on dit culturelles. En effet, de telles dynamiques offrent à leurs acteurs une grande variété de référents identitaires et ouvrent ainsi aux stratégies individuelles et collectives le possible d'une pluralité d'identifications qui s'alimentent à une large gamme de critères (ceux de l'origine, de la couleur, de la langue, de la citoyenneté, voire, dans certains cas, de la confession religieuse). Des identifications changeantes qui peuvent se combiner ou s'opposer, de manière variable selon les enjeux par rapport auxquels il s'agit pour les individus ou les groupes de se situer, rendant ainsi ambiguës, voire contradictoires, lesdites démarcations et appartenances ${ }^{8}$. Le constat de cette relativité et de cette labilité des différenciations culturelles ne doit donc pas seulement conduire à ce que les auteurs de l'Eloge de la Créolité ont appelé « la conscience non totalitaire d'une diversité préservée » (Bernabé et al., 1989, p. 28), à une acceptation même élogieuse de la différence, mais à reconnaître que l'altérité est fondamentalement constitutive de l'identité. Et qu'en conséquence, si l'on admet donc avec Stuart Hall que «L'Autre fait partie intégrante du Même, [que] l'Autre ne peut être connu que de la place où se tient le Même, [que] le Même est inscrit dans le regard de l'Autre. Et [que] ces remarques brisent les frontières entre l'Extérieur et l'Intérieur, entre ceux qui appartiennent au groupe et ceux qui n'y appartiennent pas » (Hall, 1991 , p. 48, notre traduction), alors on ne saurait accorder une existence absolue, en soi, aux frontières qui séparent les groupes ni, bien sûr, aux groupes eux-mêmes. Mais on devrait considérer, au contraire, que les premières comme les seconds, sont des constructions qui n'existent effectivement qu'en fonction des stratégies sociales et politiques de différenciation (à la fois de fission et de fusion, d'opposition et d'alliance) qui les constituent. Des démarcations et des communautés toujours imaginées, c'est-à-dire non point irréelles mais dont la réalité sociale est l'œuvre de l'imagination, au sens large, des hommes qui les font exister (voir Anderson, 1983).

\footnotetext{
${ }^{8}$ Parce que, comme le note un politologue américain, «more often than not, the various attributes of our identities do not line up the same way on any given issue, and frequently loyalties to such identities as race, religion, ethnicity, gender, class, profession, or region are divided or even in inner-conflict". Voir Adler F.H., 1995, p. 2.
} 
Ainsi le fait qu'aussi loin que les peuples de la Caraibe remontent dans leur passé, ils ne peuvent pas s'abreuver à une source unique mais qu'ils se trouvent déjà plongés dans les eaux, pour eux primordiales, de la relation planétaire ouverte dans le bruit et la fureur par la colonisation du Nouveau Monde et par les trafics du commerce triangulaire, le fait qu'ils soient donc contraints à "faire l'économie d'une Genèse » (Glissant, 1981, p. 251) et condamnés au mélange incessant de diversités renouvelées, pourrait les protéger plus que d'autres des pièges que tendent toujours la recherche de la pureté d'une origine et le souci de l'authenticité. . Parce que la singularité susdite de leur présence au monde relativise chez eux l'importance de l'enracinement dans un arrière-pays culturel préexistant (qui leur est pourtant tout aussi nécessaire qu'il l'est aux autres peuples). Que davantage qu'à s'enfermer dans l'orthodoxie d'une identité figée et close sur elle-même comme une essence, elle les pousse plutôt à se laisser emporter par des dynamiques d'identifications changeantes, surgies dans le jeu des relations avec d'autres peuples. Et qu'elle devrait ainsi leur permettre de «fréquenter leurs "valeurs" non plus comme absolu de référence mais comme modes agissants d'une relation », car «le renoncement aux pures valeurs d'origine ouvre sur un sens inédit de la mise en rapports » (Glissant, 1981, p. 30).

Ainsi, s'il y a pour eux la même nécessité que pour les autres de s'approprier leur passé pour mieux vivre leur présent et mieux penser leur avenir, les données singulières des dynamiques historiques de leurs sociétés font donc qu'il y aurait moins de risques dans ces sociétés qu'ailleurs de voir cette nécessité déboucher sur un fondamentalisme qui

\footnotetext{
${ }^{9}$ Ce n'est pas dire que, dans la Caraibe, les discours identitaires - par exemple, dans le cas des îles de colonisation française, celui de la Négritude comme celui de la Créolité ont toujours évité les omières du fondamentalisme, c'est-à-dire d'une pensée qui fait sa norme essentielle de la prescription de conformité à une origine (origine que, par ailleurs, elle reconstruit largement - à travers des manipulations de la généalogie historique effective - en fonction d'intérêts idéologiques actuels) et qui considère la soidisant authenticité comme le critère suprême de l'évaluation des expressions culturelles. Une analyse attentive de ces discours prouve qu'ils en sont souvent loin (voir Giraud, 1997), mais c'est indiquer que, plus qu'ailleurs, ils résistent mal à l'examen de la réalité des dynamiques sociales et culturelles des pays dont ils parlent.
} 
enfermerait les constructions identitaires immanquablement nourries par le travail historique dans les sectarismes des généalogies exclusives et des clôtures communautaires dont on sait qu'ils mettent aujourd'hui à feu et à sang de trop nombreux points du globe.

\section{LES EMPECHEMENTS DU NATIONALISME DANS LA CARAÏBE}

Le sentiment de la relativité des enracinements que nous avons évoqué, qui est aussi celui de la nature relationnelle des identités, fait également obstacle au développement d'une pensée forte de la nation dans les pays de la Caraïbe. En effet, la mise à distance du modèle généalogique de l'identité que consacre un tel sentiment, le peu de goût pour les manipulations de la filiation qu'il manifeste et que traduit, par exemple, le fait que, dans le monde caraïbéen, la mémoire des généalogies s'épuise le plus souvent rapidement au-delà de la génération des grands-parents, s'accommodent mal d'une problématique qui prend comme l'indique l'étymologie même du terme de nation (même si philologie n'est pas raison) - la naissance pour principe. Davantage qu'à " tempérer l'intolérable propre aux nationalismes sans les aliéner » (Glissant, 1981, p. 249), il nous semble que c'est plutôt à rendre inadéquate la problématique nationaliste elle-même que conduit, en définitive, ce sentiment. Parce que plutôt qu'à faire de l'ascendance commune, donc de la nationalité, le fondement de l'exercice des droits et du respect des devoirs politiques, il appelle ceux qui ont été réunis par l'histoire à partager et à approfondir ces droits et devoirs dans un vouloir vivre ensemble transcendant la diversité de leurs origines, donc dans une citoyenneté ouverte à tous.

Certes, dans les possessions britanniques de la Caraïbe, des mouvements nationalistes ont vu le jour, qui, dans la plupart des cas, ont conduit victorieusement, il y a une trentaine d'années, à l'avènement de nouveaux Etats. Mais cette évolution, davantage qu'elle ne vient démentir l'idée que le monde caraïbe constitue un milieu peu favorable à l'épanouissement de sentiments nationaux, tient pour beaucoup à ce qui est dit être le pragmatisme du colonialisme anglais, qui s'est toujours bien moins soucié que son équivalent français, par exemple, d'habiller la 
défense de ses intérêts matériels du manteau idéologique d'une mission civilisatrice et assimilatrice de la colonisation et de justifier cette dernière par quelques réalisations de développement social ${ }^{10}$. Toutes choses qui, lorsqu'il s'est agi pour les peuples colonisés des Antilles britanniques d'assurer un succès durable à leurs luttes en vue d'une promotion sociale effective, ne leur laissaient guère d'autre choix que celui de l'indépendance politique de leurs pays. Et encore, comme le suggèrent certains auteurs (voir Dahomay, 1993, p. 32), on peut s'interroger sur le fait de savoir si les Etats qui ont émergé de cette indépendance sont nationaux (un caractère qui est également dénié à l'Etat haïtien par certains analystes $\left.{ }^{11}\right)$.

Par contre, le caractère assimilationniste marqué du colonialisme français a ouvert une tout autre perspective aux « vieilles colonies 》 de la France $^{12}$. Là, c'est, d'une certaine manière, la même dynamique qui conduit à l'abolition de l'esclavage en 1848 et qui aboutit à la transformation, par la loi du 19 mars 1946, de ces possessions en départements de la République française. En effet, les peuples de la Guadeloupe, de la Guyane et de la Martinique, dès le moment où ils se libéraient des chaînes de la servitude et jusqu'à aujourd'hui, dans leurs efforts de remédier aux pesanteurs d'une dépendance renforcée, ont, dans leur immense majorité, toujours revendiqué l'instauration d'une égalité parfaite dans la transversalité de l'ancienne relation rénovée plutôt que

\footnotetext{
${ }^{10}$ Voir, pour une analyse comparative des deux colonialismes en question dans la région caraỉbe, l'ouvrage classique d'Arvin Murch, Black Frenchmen (1971), qui reste une bonne source d'informations, en dépit des critiques qui lui ont été adressées (dont certaines, inspirées par un parti pris nationaliste simplificateur - voir de Vassoigne, 1973 - ont pourtant été relayées par des analystes plus mesurés, comme c'est le cas de Richard Burton - voir Burton, 1978, p. 44).

11 Pour une sévère critique du nationalisme haïtien décrit comme fondamentalement «artificiel » et totalement instrumentalisé par les classes dominantes et les couches dirigeantes du pays, voir Hurbon, 1993 et Dahomay, 1993.

12 Ainsi, comme le fait observer Jean Crusol (Crusol, s.d., p. 4), si les Antilles ont été, en définitive, intégrées à leur métropole coloniale, à l'inverse des autres possessions européennes de la Caraïbe, c'est aussi parce que la possibilité leur en a été offerte, ce qui n'a pas été le cas des colonies de la Grande-Bretagne et des Pays-Bas, deux pays qui ne sont pas « inspirés par l'idée de "République Universelle" ».
} 
l'avènement d'une souveraineté nationale dans la verticalité d'une séparation d'avec la Métropole ${ }^{13}$. Une souveraineté nationale dont, leurs petits pays étant ce qu'ils sont et le rapport des forces du monde étant ce qu'il est, ils doutaient qu'elle puisse jamais être autre chose qu'illusoire, et ce avant même que le bilan des indépendances de nombre des pays voisins ne vienne apporter de l'eau au moulin de leurs réticences.

${ }^{13}$ Sur ce point, nous rejoignons tous ceux qui soulignent que les révolutions antiesclavagistes dans la Caraỉbe ont été initialement inspirées par une problématique qui est celle des droits de l'Homme davantage que celle de la souveraineté des nations. C'est ainsi que Guy Alexandre écrit : "dans son affrontement aux Français de SaintDomingue, ce n'est pourtant pas tant à la France en soi qu'à la France colonialiste et esclavagiste que la nouvelle identité haïtienne est en opposition. Il est significatif, de ce point de vue, que jusqu'au 18 mai 1803, c'est en arborant le drapeau tricolore que les troupes indigènes se lancent à l'assaut du corps expéditionnaire français, sans compter le fait que, pendant encore longtemps, ce sera en chantant "La Marseillaise". En cela, le peuple haïtien naissant, dans son identité nouvelle, participe d'un mouvement que connaîtront plus tard les peuples d'Amérique Latine, d'Afrique et d'Asie en lutte pour leur émancipation: le retournement des valeurs idéologiques et morales les plus positives élaborées par les courants de progrès - les avant-garde - des métropoles contre les politiques et pratiques déshumanisantes des mêmes métropoles. De ce point de vue, il vaut aussi la peine d'encore souligner que l'identité nationale haitienne émergeant entre la fin du XVIII ${ }^{\text {ème }}$ siècle et le début du XIX ${ }^{\text {ème }}$ siècle apparaît comme un être historique d'une absolue et radicale "modernité". Tant il est vrai que, dans le mouvement même de son émergence, cette identité nationale est toute entière articulée au combat contre le colonialisme, contre l'esclavagisme. Et, en cette demière instance, pour les droits de la persomne ». Voir Guy Alexandre, «Relations haïtiano-dominicaines et identités des deux peuples de Quisqueya », Descubrimiento, Ciencia y Arte, Santo Domingo, noviembre 1993.

Là encore, ce n'est pas dire qu'il n'existe pas aujourd'hui une pulsion nationaliste aux Antilles françaises, notamment dans les milieux intellectuels et petits-bourgeois, mais c'est simplement affirmer que les succès auxquels parviennent les forces politiques qui s'en réclament, comme celui du principal parti indépendantiste martiniquais aux dernières élections législatives et régionales, témoignent davantage d'une demande de citoyenneté accrue que d'une volonté de sécession: «the electoral success of the Mouvement Indépendantiste Martiniquais is specially important since the chances to access political sovereignty that it calls of its wishes and thus to impose a political identity in perfect congruence with the proclaimed cultural identity, seem to be moving away. Consequently, the MIM is doomed to play a simple role of protest movement". Voir Daniel, 1997, pp. 17-18. 
Sans que, contrairement à ce qui a été trop souvent dit, ces peuples ne consentent pour autant à payer leur choix de l'assimilation politique à la France au prix du reniement de leur personnalité propre; ainsi Aimé Césaire a assuré la "départementalisation》 des colonies américaines de la France tout en chantant la négritude de leurs peuples, sans qu'il ait vu l'ombre d'une contradiction dans ce qui apparaît encore aujourd'hui à certains comme un paradoxe. Tant il est vrai que la revendication de l'égalité n'exclut pas nécessairement l'affirmation de particularités mais qu'au contraire elle la suppose dès lors que ces particularités sont dévalorisées, le dénigrement de la culture d'un peuple ou d'un groupe ne laissant, en effet, à celui-ci d'autre possibilité pour parvenir à l'égalité qu'il revendique que de faire connaître la dignité de son identité bafouée. Dès lors, ce qui est souvent entendu comme la proclamation d'une différence est en définitive, dans la plupart des cas, la revendication d'un traitement égal pour tous, « un processus de mise en forme ethnique d'une demande égalitaire de participation formulée au nom de la différence » (Constant, 1996a, p. 119) ${ }^{14}$, qui implique logiquement une exigence d'universalité (même si ceux qui formulent une telle revendication ne sont pas toujours conscients de cette manipulation). Déjà Césaire, exprimant son rapport à la négritude, disait

14 «Michael Walzer a souligné avec force combien les affirmations identitaires sont avant tout des mises en forme ethniques et/ou culturelles de revendications fondamentalement intégratives. Dans la plupart des cas, c'est le refus de la majorité de tenir compte des aspirations des minorités qui conduit ces dernières à des manifestations allant parfois jusqu'au projet sécessionniste [...] le phénomène multiculturaliste s'est nourri des déficits de citoyenneté. Loin d'une volonté de "désunion nationale", la revendication multiculturaliste charrie toujours des demandes égalitaires formulées au nom de la différence. On retrouve là des mécanismes stratégiques de retournement des stigmates sociaux par lesquels des groupes érigés en communautés transforment les contraintes liées à leur appartenance à des collectivités particulières en ressources pour obtenir la garantie du respect de leurs droits. Car plus grande est la crainte des minorités de se voir interdire l'accès aux ressources étatiques, plus forte est leur tendance à resserrer leurs liens internes; moins la société tend à satisfaire leurs demandes de reconnaissance par des mesures d'accommodement, plus elles tendent à manipuler des marqueurs symboliques peu ou prou imaginaires. C'est pourquoi on a assisté à une politisation spectaculaire des réclamations identitaires ». Voir Fred Constant, 2000, pp. $45-46$. 
que s'il s' " exige bêcheur de cette unique race, [...] c'est pour la faim universelle, pour la soif universelle » (Césaire, 1956, p. 28) et Sartre surenchérissait en déclarant que le Nègre « est celui qui vit jusqu'au bout le particularisme pour y trouver l'aurore de l'universel » (Sartre, 1948, p. XLII). Ainsi, en cherchant à acquérir la citoyenneté de la France, les peuples de la Guadeloupe, de la Guyane et de la Martinique ne visaient pas, dans leur grande majorité, à s'approprier l'identité culturelle française mais à supprimer l'injustice coloniale en se donnant les moyens de bénéficier, sur un pied d'égalité avec les autres Français, des droits que cette citoyenneté est censée garantir.

L'évolution des possessions françaises de la Caraibe n'est cependant pas aussi singulière qu'elle peut le paraître superficiellement, comme en témoigne l'histoire politique récente de territoires comme Porto Rico, étroitement associé aux Etats-Unis, ou Curaçao, fortement intégré dans le royaume des Pays-Bas, où des référendums ayant eu lieu en novembre 1993 n'ont accordé que $4 \%$ des voix, dans le premier cas, $0,5 \%$ dans le second, au vote indépendantiste. En décembre 1998, ce vote est même tombé à $2,3 \%$ à Porto Rico. Et maints analystes affirment que les dynamiques politiques de ces territoires en particulier et de la Caraỉbe en général ne peuvent plus être pensées en fonction de la seule opposition entre nationalisme et colonialisme. Ainsi, les directeurs d'un ouvrage récemment paru sur la situation portoricaine affirment: "In Puerto Rico, it has been possible for a significant number of the population to imagine the "nation" without a "Puerto Rican" state and to view the United State's state apparatus as a more beneficial structure to advance the "nation's" interests" (Grosfoguel et al., 1997, p. 17) et rencontrent par-là même le point de vue d'un des spécialistes les plus avertis de l'étude des possessions néerlandaises de la Caraïbe, qui écrit : "By the early 1990s, opting for the prolongation of a postcolonial liaison had become a thoroughly respectable means of exercising the right to self-determination" (Oostindie, 1996, p. 232).

Ces dynamiques apparaissent d'autant plus contraires à ce que voudrait la logique traditionnelle du nationalisme que parfois elles sont allées à l'encontre du souhait initial de la puissance coloniale concernée 
de "passer la main » et débouchent même, dans certains cas, sur un renforcement de la demande d'une intégration politique complète à la Métropole. Ainsi, Gert Oostindie rappelle justement, dans le texte déjà cité, que l'indépendance du Suriname a été, pour une large part, imposée par les Pays-Bas, qu'après que celle-ci eut été proclamée ce sont les autorités elles-mêmes du nouvel Etat qui se sont opposées à ce que la responsabilité du traitement externe de la crise surinamienne soit transférée des Pays-Bas aux organisations internationales, comme le souhaitait le Gouvernement de La Haye, et qu'enfin le territoire d'Aruba n'a pas eu, en définitive, à payer, pour sa sortie de la Fédération des Antilles néerlandaises, le prix de l'indépendance politique qu'au départ le pouvoir néerlandais lui réclamait d'acquitter contre le sentiment des responsables de ce territoire. Au point que notre collègue hollandais évoque ailleurs le "spectre d'une recolonisation" de la Caraibe néerlandophone (voir Oostindie, 1992) ${ }^{15}$. De même, pour ce qui concerne Porto Rico, c'est le Congrès des Etats-Unis qui constitue aujourd'hui le principal obstacle à la satisfaction de l'exigence, devenue majoritaire dans l'île, que celle-ci devienne un nouvel Etat de l'Union.

Dans ces conditions, les évolutions politiques en question ne peuvent pas être considérées comme représentant un triomphe de l'aliénation coloniale mais une consécration de l'aptitude de peuples hier encore complètement assujettis à conquérir un espace de liberté et une possibilité de promotion à l'intérieur du système qui les domine. Quelque ironie qu'il y ait à devoir reconnaître aujourd'hui un tel paradoxe, comme le souligne Ramón Grosfoguel : «Una de las grandes ironias historicas del Caribe es la siguiente : las repúblicas caribeñas que emergieron en lucha contra el control imperial, en busca de mayor soberania y libertad, hoy tienen menos poder de maniobra en el sistema-mundial capitalista,

\footnotetext{
${ }^{15}$ Ainsi, Gert Oostindie rappelle qu'un groupe de Surinamiens expatriés de grand renom fit paraître en 1993 un manifeste demandant l'organisation d'un référendum sur un réaménagement des relations entre les Pays-Bas et le Suriname qui ramènerait, dans une large mesure, celles-ci à ce qu'elles étaient avant la proclamation de l'indépendance de ce dernier pays; un référendum dont notre collègue précise que l'opposition des autorités néerlandaises à sa tenue rend celle-ci tout à fait improbable.
} 
mayor inestabilidad en la práctica de la democracia y más bajos niveles de vida que las islas que mantuvieron lazos con las metrópolis" (Grosfoguel, 1996) ${ }^{16}$.

L'ambiguïté fondamentale des évolutions politiques récentes dans la Caraỉbe conduit donc nombre de Caraỉbéens à revendiquer pour euxmêmes à la fois les bénéfices conférés par la citoyenneté d'une grande puissance dont le centre se situe hors de leur région et les avantages de dispositions sociales et politiques particulières que légitimerait leur identité culturelle propre. Cette ambiguité - dont on peut prendre pour emblème le mot d'ordre «le meilleur des deux mondes» du Parti Progressiste Démocratique portoricain ou le penchant que les couches populaires des Antilles françaises auraient pour «des pratiques plurielles que l'unitarisme idéologique du nationalisme ne pouvait que manquer » (Dahomay, 1993, p.57) - a bien évidemment partie liée avec la particularité profonde de l'expérience historique créole que nous avons rappelée. Ainsi, il fait sens de souligner que «Peoples and cultures that have been coerced into more than one history through domination, slavery, and colonialism have the ethico-political need and authority to make their presence felt in all these histories. Ambivalence gives these cultures a double directionality: a here or the present home, and a there or the elsewhere in terms of which metropolitan contemporaneity can be interrogated and transformated" (Rajagopalan Radhakrishnan, cité in Grosfoguel et al., 1997, pp. 17-18) et de préciser que cette ambivalence donne à ces peuples - et pas seulement dans le cas, qu'envisage l'auteur de cette citation, où ils sont en diaspora - «the potential to represent the

\footnotetext{
16 Jean Crusol en vient, de son côté, à la même conclusion: «Lorsqu'on prend aujourd'hui une vue de la période qui nous sépare de 1946, on est forcé de constater que les progrès réalisés par la Martinique et les autres DOM, sont bien plus impressionnants que ceux réalisés par des îles comparables mais indépendantes dans la Caraíbe, l'Océan Indien ou le Pacifique. Les progrès économiques sont plus importants, les inégalités sociales moins fortes, la couverture sociale meilleure, l'éducation et la santé plus développées, la démocratie et les droits de l'homme mieux assurés [...] En Jamaïque, à Trinidad, au Guyana les taux de chômage sont supérieurs à $20 \%$, mais à la différence de la Martinique, il n'existe pas de soutien aux chômeurs, et les salaires sont 5 à 10 fois plus faibles que chez nous » (Crusol, s.d., p. 4).
} 
third world within the first world not through easy assimilationism or strategic opportunism, but through a fundamental questioning of the manner in which dominant regimes play and dictate the identity game to subaltern groups on the basis of a rigged and stacked text" (Id.)

\section{ET POURTANT, DES EXCLUSIVISMES IDENTITAIRES A REDOUTER}

La Caraïbe n'est pas un paradis de la raison. Bien qu'il pourrait être attendu que - compte tenu des conditions de la formation des sociétés de cette région - les populations caraỉbéennes vivent leur radicale bâtardise loin du pathos de la recherche de quelque pureté originelle que ce soit, les revendications identitaires qui s'y développent trébuchent souvent sur les pièges que leur tend l'exclusivisme du modèle généalogique de l'identité. Les sociétés caraïbéennes sont donc, elles aussi, « travaillées » par des conceptions essentialistes de l'identité qui, par le potentiel d'intolérances et d'exclusions qu'elles contiennent, font peser de graves menaces sur la vie sociale et politique des pays considérés. Les dérives racialistes ou communautaristes que l'on peut observer dans nombre de sociétés de la région et les tensions « interraciales » ou interethniques dont ces dérives sont l'occasion (comme cela est, entre autres exemples, régulièrement et fortement le cas au Guyana, au Suriname ou à Trinidad) ${ }^{17}$, phénomènes que vient parfois renforcer le rejet de populations migrantes de la Caraïbe (comme on peut l'observer, par exemple, en République Dominicaine ou dans certains départements français d'Amérique), témoignent de la réalité de ces menaces. Des

\footnotetext{
${ }^{17}$ Pour un aperçu rapide mais relativement complet de ces tensions, voir, par exemple, Premdas, 1996, pp. 20-21. Cet auteur juge que lesdites tensions pourraient finir par déstabiliser la région et ajoute: "If this should occur, one can expect the worst, including refugees flows and the involvement of overseas Caribbean populations and their allies in the fray". Des tensions qui conduisent Fred Constant à juger, de son côté, que, dans les sociétés en question, "Opposition et majorité, leaders afro-antillais et indo-antillais manifestent la même impuissance à produire une vision de l'intérêt national susceptible d'échapper aux schémas caricaturaux de l'affrontement intercommunautaire. L'orientation de l'action publique est invariablement reformulée dans le langage explosif des relations intercommunautaires »(Constant, 1996b, p. 120).
} 
phénomènes qui, de plus, ne sont pas étrangers au maintien de la fragmentation de la région que nous évoquions pour commencer.

C'est qu'en l'absence d'une vision largement et profondément partagée des grands principes et des règles primordiales qui doivent régir le jeu social et politique ${ }^{18}$, qui s'enracinerait elle-même dans l'adhésion commune à un corps de valeurs intégrant et transcendant les systèmes normatifs des groupes particuliers, les sociétés - quelles qu'elles soient sont livrées sans défense aux effets désintégrateurs des forces centrifuges que nourrit la volonté de chaque groupe de réaliser des aspirations qui lui sont propres, y compris par la captation des moyens de l'Etat qui sont théoriquement à tous. Parce qu'alors les conditions du pouvoir et donc du vouloir vivre ensemble ne sont pas pleinement réunies. Or, dans nombre de sociétés caraïbéennes, en particulier celles qui - selon une définition désormais classique - sont dites plurales ${ }^{19}$ (comme c'est le cas des formations sociales de la partie méridionale de l'arc caraïbe dont nous évoquions plus haut les divisions), une telle vision a du mal à s'imposer, précisément du fait du pluralisme culturel qui divise ces formations. Et ce d'autant que les effets de ce pluralisme n'y sont pas tempérés, comme nous l'avons déjà dit, par un puissant sentiment national. Dès lors, le libre jeu des particularismes, même s'il est limité par le principe abstrait du respect des différences, provoque des affrontements destructeurs entre les diverses communautés en présence ainsi que de violents conflits de légitimité qui bloquent gravement le fonctionnement social, car-comme le souligne justement le sociologue britannique d'origine jamaïcaine Harry Goulbourne - «People sharing a common territory, but holding a variety of moral and ethical precepts embedded in cultural norms, are

${ }^{18}$ Des principes qui, pour autant, ne font pas disparaître les oppositions et les conflits d'intérêt mais qui les régulent.

${ }^{19}$ "A plural society is a culturally divided society in which each cultural section has its own exclusive way of life, with its own distinctive system of action, ideas and values, and social relations". Voir Smith, 1960, p. 768. 
likely sooner or later to become antagonistic when the only thing they are presumed to share is their difference" (Goulbourne, 1992, p. 368) ${ }^{20}$.

C'est donc en définitive toutes les formules prétendant aligner l'organisation politique jugée légitime sur des appartenances culturelles prétendues authentiques (que ces formules se réclament d'un nationalisme monoculturel à prétention hégémonique ou du modèle multiculturaliste) qui apparaissent problématiques. Et ce d'autant plus que cet alignement repose le plus souvent sur une conception essentialiste de l'identité culturelle individuelle, assimilant celle-ci à une donnée de nature, à un atavisme, une hérédité, alors que cette identité est fondamentalement l'expression d'un choix et le fruit d'une reconstruction de la part de l'individu : les «racines » d'un individu ne sont les siennes que dans la mesure où il les reconnaît comme telles et où il les fait telles (voir Giraud, 1987). Dans l'optique de cette vision naturalisante de l'identité individuelle, l'aplatissement de l'appartenance politique sur l'appartenance culturelle revient à confondre ce qui doit être rigoureusement distingué : le domaine de l'être et le règne de la valeur, le fait et le droit. Et la citoyenneté, qui est en son principe l'expression d'une exigence et d'un projet, qui manifeste un vouloir vivre ensemble, une volonté d'appartenir à un système politique, est alors réduite à n'être plus qu'un état de fait, la marque de la contingence d'une naissance.

Une telle réduction du politique au culturel ou, si l'on préfère, au national est particulièrement néfaste en ce qu'elle est génératrice d'exclusions, comme en témoigne, par exemple, la situation qui est faite, dans certains pays de la Caraỉbe (et ce à l'instar de ce qui est observé un peu partout dans le monde en ce qui concerne les immigrés), aux populations venues, en nombre croissant, d'autres pays de l'archipel (voir Giraud, 1999). Ainsi, alors qu'on note aujourd'hui dans bien des pays de

20 «L'affirmation d'une identité culturelle est toujours en elle-même une source potentielle de conflit, voire de totalitarisme. Une culture imaginée comme «authentique » se définit par opposition à des cultures voisines en les appréhendant comme radicalement différentes; cette supposition d'altérité équivaut à un principe d'exclusion dont la conclusion logique est une opération de purification ethnique $\gg$. Voir Bayart, 1996-1997. 
la Caraïbe une tendance renforcée à vouloir fonder la communauté politique sur une prétendue cohérence culturelle ou, comme le dit Justin Daniel, à vouloir œuvrer à «une illusoire superposition de l'identité culturelle et de l'identité politique»-au point que les discours identitaires tendent à $\mathrm{y}$ absorber la totalité de la revendication politique $^{21}$ - il convient donc, à l'inverse, de décrocher l'action politique de la soumission aux filiations culturelles et aux allégeances communautaires. Et ce pour asseoir solidement cette action sur un corps de principes qui, porteur d'une visée d'universalité et transcendant ainsi les systèmes normatifs de groupes particuliers ${ }^{22}$, serait tourné vers

${ }^{21}$ Cela est particulièrement vrai aux Antilles françaises où « D'un nationalisme pur et dur, on est passé à une problématique de la créolité qui est avant tout une revendication de l'identité culturelle où la politique stricto sensu occupe une place restreinte. D'où la difficulté actuelle des théories de l'identité antillaise à poser effectivement la question du pouvoir et de l'autorité politique. D'où aussi l'aspect incantatoire de telles idéologies ayant du mal à s'enraciner dans une pratique politique concrète. C'est désormais l'art [...] qui vient paradoxalement occuper la place laissée vacante par une politique nationaliste concrète ». Voir Dahomay, 1993, p. 56. Voir également Giraud, 1997.

${ }^{22}$ Il convient ici de préciser que - dans l'interprétation du concept d'universalité que nous retenons - l'universalité, tout en dépassant les particularismes culturels, n'est pas indifférente à ceux-ci, parce que : «Le concept d'universalité n'est pas synonyme de neutralité vis-à-vis de certaines valeurs et certains modes de vie mais fait plutôt référence, en première instance, aux métanormes de réciprocité symétrique qui doivent agir en tant que principes régulateurs et guider les processus discursifs de résolution des conflits et, en seconde instance, aux normes et principes reconnus par ceux qui sont susceptibles d'en être affectés [...] En conséquence, l'universalité est un principe régulateur d'un processus discursif de résolution des conflits [entre traditions culturelles différentes], dans lequel et par lequel les participants discutent ensemble des valeurs, des principes et des besoins à satisfaire qui méritent d'être institutionnalisés en tant que normes communes. Ce n'est donc pas l'existence d'un individu désincarné, atomisé, [...] qui fonde cette approche. Assumant le fait que les identités individuelles et collectives sont acquises par des processus de socialisation complexes, qui impliquent à la fois d'intérioriser des normes et des traditions sociales et de développer des capacités de réflexion et de critique vis-à-vis des normes, principes et traditions, la conception qui constitue le cour de cette théorie est une conception intersubjective et interactive de l'individualité et de l'autonomie. Elle est donc capable d'adopter les "explications » communautaristes du noyau social de la nature humaine, sans pour autant abandonner les idées d'universalité et de droit moral [...] C'est sur ce terrain que nous apprenons à élaborer des compromis, à prendre un recul réflexif sur notre propre perspective, à 
l'accomplissement de l'idéal démocratique de l'égalité de tous les citoyens. C'est qu'en effet, «la démocratie requiert une [...] ontologie fondée sur l'abîme qui sépare l'être du devoir être » (Dahomay, 1993, p. 52).

La Caraïbe - tout en n'étant pas à l'abri de ce que nous venons d'appeler les exclusivismes identitaires - a cependant, comme il a été dit précédemment, plus que d'autres régions du monde, les moyens du décrochage en question. Notamment parce qu'elle a été le lieu d'une expérience historique, souvent présentée sous l'image du «laboratoire créole », à travers laquelle - par delà les souffrances et les violences qui l'ont marquée (colonisation, génocide, traite négrière, esclavage, racisme, exploitation et dépossession) - les hommes et les femmes de la région peuvent connaître et reconnaître aujourd'hui, plus clairement qu'ailleurs, que l'émergence ou la consolidation d'un ordre démocratique passe, dans une tension vers l'universel des droits de l'Homme, par l'assomption de la contingence, de la complexité et de la relativité des appartenances culturelles. Et non par le souci de la conformité à une origine, qui nourrit les clôtures communautaires.

\section{TENIR LES DEUX BOUTS DE LA CHAINE}

L'autonomie qu'il convient de garantir à la construction politique conduit ainsi - en matière d'identité - à n'accorder de valeur, au-delà de la diversité des origines de tous ceux qui sont partie prenante de cette construction, qu'à l'expérience - partagée par les enfants de l'esclavage et par les rejetons du travail sous contrat - des souffrances qui ont été imposées à chacun de ces groupes et des luttes qu'ils ont dû mener pour tenter d'en finir avec celles-ci. Et, ce faisant, à n'avoir d'autre fidélité au passé que négative, celle-là même qui fait s'écrier : «plus jamais ça, sous une forme ou sous une autre!»

reconnaître et recréer ce que nous avons en commun, et que nous parvenons à déterminer celles de nos traditions qui valent d'être conservées, abandonnées ou modifiées ». Voir Cohen et Arato, 1993, pp. 64 et 66. 
C'est dire qu'il ne s'agit pas tant pour les héritiers des expériences créoles de renoncer à l'utilisation stratégique des identités, au jeu subtil des relations et des affirmations d'identité qu'appellent les situations dans lesquelles leurs actions s'inscrivent - qui le pourrait ? - que de se tenir sur leur garde face au péril de la substantialisation de ces identifications en des essences identitaires, de leur absolutisation en des atavismes qui sont autant de carcans et d'excuses, en ne perdant jamais de vue que la démocratie à construire est pour tous.

Il n'est donc pas question, dans nos vues, que les populations caraibéennes jettent par-dessus bord la particularité de leurs diverses expériences historiques et l'originalité de leurs différentes productions culturelles au motif que la problématique de l'authenticité par laquelle on tente souvent d'en rendre compte est inadéquate à leur réalité profonde. Ce serait là une attitude intellectuellement injustifiée et politiquement inconséquente. Il s'agit plutôt de «tenir les deux bouts de la chaîne ", comme nous y invite Stuart Hall lorsqu'il écrit à propos des " expériences noires»: "attempting to valorize and defeat the marginalization of the variety of Black subjects and to really begin to recover the lost histories of a variety of Black experiences, while at the same time recognizing the end of any essential Black subject" (Hall, 1991, p. 57). Donc de veiller à ce que les particularités qui nous occupent ici ne soient pas enfermées dans des substances identitaires qui réifient les dynamiques sociales et culturelles dont elles sont faites (enfermement qui serait tout aussi détestable que celui dans lequel les civilisations dominantes ont depuis longtemps tenté de les confiner, en s'en démarquant). De même, tant il est vrai que "Identity is not in the past to be found, but in the future to be constructed" (Hall, 1995, p. 12), il faut que chaque groupe se soucie de ce que la nécessaire réappropriation qu'il a à effectuer du passé dont il porte encore les marques mais dont il n'est cependant pas prisonnier - et qui doit être menée en fonction des enjeux du présent - ne se transforme pas en une fixation passéiste qui clôturerait l'avenir, qui est toujours invention et non répétition.

Tout cela implique de considérer les identités comme 1'expression d'une incessante créativité qui - quoique s'exerçant à partir des 
sédimentations culturelles qui leur préexistent et qui en partie les contraignent - ne s'embarrasse pas du respect d'une quelconque orthodoxie mais témoigne d'une liberté et d'une multiplicité d'identifications, celles-là même que loue Edouard Glissant quand il affirme qu'il y a lieu de revenir «non pas à une outrance renouvelée de spécificités mais à une liberté totale [...] de leurs rapports, frayée au chaos même de leurs affrontements » (Glissant, 1990, p. 75). Identifications qui sont en définitive motivées par les enjeux sociaux et politiques des situations diverses, notamment des luttes, dans lesquelles les populations considérées et les individus composant celles-ci se trouvent engagés et qui sont, par-là même, pour ces populations comme pour ces individus, relatives, variées, flexibles et changeantes.

C'est précisément cette relativité, cette diversité et cette labilité des dynamiques identitaires que tout projet démocratique dans les sociétés caraïbéennes doit prendre en compte, s'il ne veut pas rater son objet. Il doit, en effet, être " a politics which [...] is able to address people through the multiple identities which they have - understanding that those identities do not remain the same, they are frequently contradictory, that they cross-cut one another, that they tend to locate us differently at different moments" (Hall, 1991, p. 59). Alors, avec la concrétisation d'un tel projet, la Caraïbe de puissance pourrait devenir acte.

\section{REFERENCES CITEES}

ADLER F.H.

"Racism, Difference and the Right in France », Modern and Contemporary France, January 1996.

ANDERSON B.

1983, Imagined Communities, Reflections on the Origin and Spread of Nationalism, London, Verso (traduction française: L'imaginaire national, Réflexions sur l'origine et l'essor du nationalisme, Paris, La Découverte, 1996). 
BAYART J. F.

(1996-1997), «L'imaginaire dans l'affirmation identitaire » (entretien), Sciences Humaines, $\mathrm{n}^{\circ} 15$, Hors série «Identité, Identités », déc.-janv., pp. 42-45.

BOURDIEU P.

1987, «Espace social et pouvoir symbolique », in Pierre Bourdieu, Choses dites, Paris, Les éditions de Minuit, pp. 147-166.

BURAC M.

«The French Antilles and the wider Caribbean », in Richard D.E. Burton and Fred Reno (eds.), French and West Indian: Martinique, Guadeloupe and French Guiana Today, London, Macmillan, pp. 98-111.

BURTON R., D.E.

1978, Assimilation or Independence? Prospects for Martinique, Occasional Monographs Series $\mathrm{n}^{\circ} 13$, Centre for Developing Area Studies, McGill University, Montreal.

CESAIRE A.

1956, Cahier d'un retour au pays natal, Paris, Présence Africaine.

COHEN J.L. et ARATO A.

1993, «Un nouveau modèle de société civile», Les Temps modernes, n 564, juil.-août, pp. 40-70.

CONSTANT F.

1996a, "La démocratisation dans les Caraỉbes du Commonwealth: citoyenneté, ethnicité, égalité », in Laënnec Hurbon (dir.), Les transitions démocratiques, Paris, Syros;

1996b, «L'intégration régionale des Caraïbes du Commonwealth: les mythes unitaires face aux enjeux politiques internes », in Denis-Constant Martin, Les démocraties antillaises en crise, pp. 109-138;

2000, Le multiculturalisme, Paris, Flammarion, Coll. Dominos.

CRUSOL J.

s.d. La loi d'orientation et la supercherie de "Basse-Terre». Pour regarder en face les réalités $d u X X I^{i m e}$ siècle, Parti Socialiste, Fédération de la Martinique, s.l, document multicopié. 


\section{DAHOMAY J.}

1993, «Les faiblesses du nationalisme antillais. Le nationalisme entre terreur et démocratie », Chemins Critiques, vol. 3, n 1-2, déc., pp. 3170.

DANIEL J.

1997, Cultural Identity and Political Identity in the French Antilles and Puerto Rico: Myths and Realities, Communication présentée à la Conférence annuelle de 1'Association des Etudes Caraíbéennes, Baranquilla, Colombie, 26-30 mai (document multicopié).

DE VASSOIGNE $Y$.

1973, Note bibliographique sur Black Frenchmen d'Arvin Murch, Acoma, 4-5 avril, pp. 188-189.

GAUTHIER F.

1997, «Et du citoyen ! », Chemins Critiques, vol. 3, n 3, janv., pp. 190205.

GIRAUD M.

1987, "Mythes et stratégies de la "double identité" », L'Homme et la Société, $\mathrm{n}^{\circ} 83,1^{\text {er }}$ trimestre 1987, pp. 59-67;

1997, «De la Négritude à la Créolité : une évolution paradoxale à l'ère départementale », in Fred Constant et Justin Daniel (dir.), 1946-1996 Cinquante ans de départementalisation outre-mer, Paris, L'Harmattan, pp. 373-403 ;

1999, «Les migrations intracaraïbénnes : l'autre exclusion », Plein droit, La revue $d u$ Groupe d'Information et de Soutien des Immigrés, $n^{\circ} 43$ (Outre-mer, autre droit), sept., pp. 3-6;

2001, «Après la colonie, la nation? Le devenir politique des départements français d'Amérique en question », Pouvoirs dans la Caraïbe, $\mathrm{n}^{\circ} 12$, pp. 79-109.

GLISSANT E.

1981, Le discours antillais, Paris, Editions du Seuil ;

1990, Poétique de la Relation, Paris, Gallimard. 
GOULBOURNE H.

1992, «New issues in black British politics», Information sur les sciences sociales, $\mathrm{n}^{\circ} 31,2$, juin, pp. 355-373.

GROSFOGUEL R.

1996, «El Caribe y la "Independencia realmente existente" », Diálogo, mayo, p. 26.

GROSFOGUEL R., NEGRON-MUNTANER F. and GEORAS C.S.

1997, «Beyond Nationalist and Colonialist Discourses: The Jaiba Politics of the Puerto Rican Ethno-Nation », in Frances NegrónMuntaner and Ramón Grosfoguel (eds.), Puerto Rican Jam. Rethinking Colonialism and Nationalism, Minneapolis, University of Minnesota Press, pp. 11-38.

\section{HALL S.}

1991, "Old and New Identities, Old and new Ethnicities », in Anthony D. King (ed.), Culture, Globalization and the World-System, op. cit., pp. 41-68.

\section{HURBON L.}

1993, « Nationalisme et démocratie en Haïti », Chemins Critiques, vol. 3, $\mathrm{n}^{\circ} 1-2$, déc., pp. 7-30.

MURCH A.

1971, Black Frenchmen. The political Integration of the French Antilles, Schenkman Publishing Company, Cambridge, Mass.

OOSTINDIE G.

1992, «The Dutch Caribbean in the 1990s: Decolonization, Recolonization? », Caribbean Affairs, n 5-1, pp. 103-119.

1996, «Ethnicity, Nationalism and the Exodus: The Dutch Caribbean Predicament », in Gert Oostindie (ed.), Ethnicity, Social Structure, and National Identity in the Caribbean. Essays in Honor of Harry Hoetink, London, Macmillan, pp. 228-249. 
Faire la Caraïbe, comme on refait le monde

\section{PREMDAS R.}

1996, Ethnicity and Identity in the Caribbean: Decentering a Myth, Working Paper \#234, The Helen Kellog Institute for International Studies, University of Notre-Dame.

SARTRE J.P.

«Orphée noir », préface à Léopold S. Senghor (dir.), Anthologie de la nouvelle poésie nègre et malgache de langue française, Paris, PUF, 1948, pp. IX-XLIV.

SMITH M.G.

1960, «Social and Cultural Pluralism », in Vera Rubin (ed.), Social and Cultural Pluralism in the Caribbean, Annals of the New York Academy of Science, 83(5), pp. 763-770.

WAGLEY C.

1960, «Plantation-America: A Culture Sphere », in Vera Rubin (ed.), Social and Cultural Pluralism in the Caribbean, New York, Annals of the New York Academy of Sciences, pp. 3-13.

WILSON P.J.

1973, Crab Antics. The social anthropology of English-speaking Negro Societies of the Caribbean, New Haven, Yale University Press. 


\section{RESUME}

L'histoire de l'archipel caraïbe - avec les transbordements et les mélanges de populations venues des quatre continents qu'elle a connus - présente la plus forte anticipation de ce que la planète tout entière est en train de devenir: un espace où le Monde entre en collision avec lui-même. Dans un tel contexte, il est bien difficile d'évoquer ou d'invoquer une identité qui s'enracinerait dans le superbe isolement d'une généalogie immémoriale. Construire la Caraïbe comme identité ne peut qu'équivaloir à sublimer un passé de déracinements, de dépossessions et de métissages dans le vœu d'une égalité et d'une justice par tous partagées. Dès lors, il $\mathrm{y}$ a grave danger à ce que les acteurs de cette construction la soumettent à des allégeances communautaires.

\section{ABSTRACT}

The history of Caribbean archipelago - including transfer and mixture of populations from all over the four continents - gives us a strong hint of the current main global trend: a place where the whole world is colliding with itself. So, it is quite difficult to refer to and to praise an identity that would root in the splendid isolation of some beyondmemory genealogy. Building the Caribbean as an identity just means sublimation of a past made with tearing up, dispossession, and metissage, in the common hope for justice for everybody. Therefore, actors of this Caribbean identitybuilding shall never forget that nothing good may be expected from communalism-oriented policies. 